UCRL-JC-128088

PREPRINT

\title{
First Significant Image Improvement from a Sodium-Layer Laser Guide Star Adaptive Optics System at Lick Observatory
}

\author{
S. S. Olivier, C. E. Max, H. W. Friedman, J. An, K. Avicola, B. V. Beeman, \\ H. D. Bissinger, J. M. Brase, G. V. Erbert, D. T. Gavel, \\ K. Kanz, B. Macintosh, K. P. Neeb, K. E. Waltjen
}

This paper was prepared for submittal to the Society of Photo-Optical Instrumentation Engineers 1997 International Symposium on Optical Science, Engineering and Instrumentation San Diego, CA

July 27-August 1, 1997

July 14, 1997

This is a preprint of a paper intended for publication in a journal or proceedings. Since changes may be made before publication, this preprint is made available with the understanding that it will not be cited or reproduced without the permission of the author. 


\section{DISCLAMER}

This document was prepared as an account of work sponsored by an agency of the United States Government. Neither the United States Government nor the University of California nor any of their employees, makes any warranty, express or implied, or assumes any legal liability or responsibility for the accuracy, completeness, or usefulness of any information, apparatus, product, or process disclosed, or represents that its use would not infringe privately owned rights. Reference herein to any specific commercial product, process, or service by trade name, trademark, manufacturer, or otherwise, does not necessarily constitute or imply its endorsement, recommendation, or favoring by the United States Government or the University of California. The views and opinions of authors expressed herein do not necessarily state or reflect those of the United States Government or the University of Califomia, and shall not be used for advertising or product endorsement purposes. 


\title{
First significant image improvement from a sodium-layer laser guide star adaptive optics system at Lick Observatory
}

\author{
S. S. Olivier, C. E. Max, H. W. Friedman, J. An, K. Avicola, B. V. Beeman, \\ H. D. Bissinger, J. M. Brase, G. V. Erbert, D. T. Gavel, \\ K. Kanz, B. Macintosh, K. P. Neeb, and K. E. Waltjen \\ Lawrence Livermore National Laboratory, PO Box 808, Livermore, CA 94551
}

\begin{abstract}
Atmospheric turbulence severely limits the resolution of ground-based telescopes. Adaptive optics can correct for the aberrations caused by the atmosphere, but requires a bright wavefront reference source in close angular proximity to the object being imaged. Since natural reference stars of the necessary brightness are relatively rare, methods of generating artificial reference beacons have been under active investigation for more than a decade. In this paper, we report the first significant image improvement achieved using a sodium-layer laser guide star as a wavefront reference for a high-order adaptive optics system. An artificial beacon was created by resonant scattering from atomic sodium in the mesosphere, at an altitude of 95 $\mathrm{km}$. Using this laser guide star, an adaptive optics system on the $3 \mathrm{~m}$ Shane Telescope at Lick Observatory produced a factor of 2.4 increase in peak intensity and a factor of 2 decrease in full width at half maximum of a stellar image, compared with image motion compensation alone. The Strehl ratio when using the laser guide star as the reference was $65 \%$ of that obtained with a natural guide star, and the image full widths at half maximum were identical, $0.3 \mathrm{arc} \mathrm{sec}$, using either the laser or the natural guide star. This sodium-layer laser guide star technique holds great promise for the world's largest telescopes.
\end{abstract}

Keywords: adaptive optics, laser guide star, sodium layer, imaging

\section{INTRODUCTION}

Images of astronomical objects made with ground-based telescopes are blurred by variations in the index of refraction of air due to atmospheric turbulence. These aberrations limit the resolution (e.g., the full width at half maximum, FWHM) of long-exposure images to -0.5 arc sec at visible and near-infra-red wavelengths. If the effects of turbulence could be fully corrected, telescopes of diameter 1 to $10 \mathrm{~m}$ would achieve resolutions of 0.1 to $0.01 \mathrm{arc} \mathrm{sec}$ at $0.5 \mu \mathrm{m}$ wavelength. The potential improvement in resolution is a factor of 5 to 50 !

Adaptive optics systems which sense and correct atmospheric aberrations were proposed by Babcock in 1953 (1). Approximately 10 such systems are now installed on astronomical telescopes (2), including one developed at Lawrence Livermore National Laboratory (LLNL) for the $3 \mathrm{~m}$ Shane Telescope at Lick Observatory $(3,4)$. These systems use a natural star as reference beacon. However, requirements on brightness and proximity restrict natural guide star adaptive optics systems to a small fraction $(<10 \%)$ of the sky.

In principle, laser guide star systems create reference beacons anywhere on the sky and can dramatically improve sky coverage. Two ways to create a laser guide star have been pursued. The first uses Rayleigh scattering from air molecules at altitudes of $10-15 \mathrm{~km}$. A system using this technique is in operation on a $1.5 \mathrm{~m}$ telescope at the Starfire Optical Range (5).

However, since Rayleigh scattering is limited to $<15 \mathrm{~km}$ height by the exponential decrease in air density, the wavefront from a Rayleigh beacon does not sample the same aberrations as the wavefront from an astronomical object. This is called the cone effect because the Rayleigh beacon samples a cone instead of a cylinder. The cone effect, also known as focus anisoplanatism (6), is more pronounced for a lower-altitude reference beacon and a larger telescope. For telescopes with diameters of 6 to $10 \mathrm{~m}$, the cone effect from a Rayleigh beacon is a severe limitation.

Use of laser-produced resonance fluorescence of atomic sodium in the mesosphere at a height of $\sim 95 \mathrm{~km}$ (at a wavelength 589 $\mathrm{nm}$ corresponding to the $\mathrm{Na} \mathrm{D}_{2}$ line) was proposed in $1985(7,8)$. The $95 \mathrm{~km}$ height of the sodium layer leads to a significant reduction of the cone effect, compared to a Rayleigh beacon. 
High-order wavefront phase measurements using a sodium-layer laser guide star were first performed in 1992 at LLNL (9). Low-order measurements were previously made by MIT Lincoln Laboratory when laser guide star centroid motions were recorded in 2 contiguous subapertures (10). Image improvement using a sodium-layer laser guide star with a low-order imagestacking system on the Multiple Mirror Telescope was accomplished in 1994. This system allowed control of the relative tip-tilt between 2 (and subsequently, all 6) of the Multiple Mirror Telescope's $1.8 \mathrm{~m}$ primary mirrors (11). Here first significant image improvement with a high-order adaptive optics system and a sodium-layer laser guide star

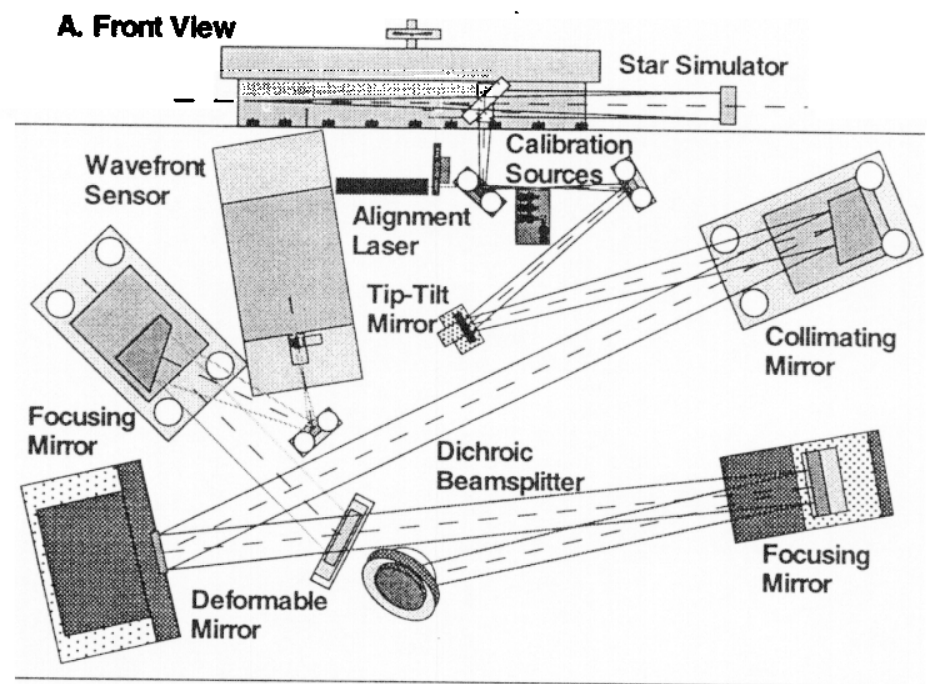

B. Back View

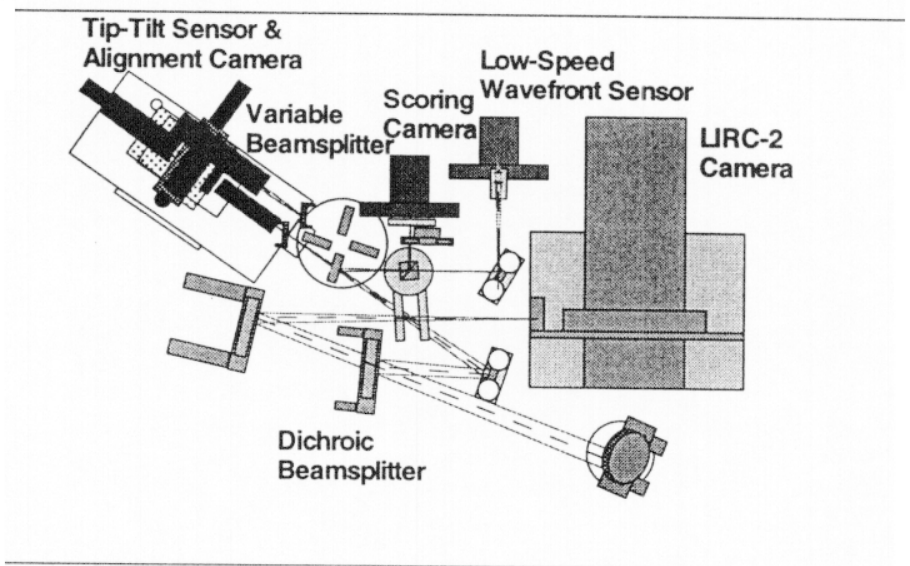

Figure 1. Lick adaptive optics system schematic. (A) Light from the telescope enters from top e ter on the diagram and hits two turning mirrors, the tip-tilt mirror, an off-axis parabolic collimating mirror, 1 then the deformable mirror. The light then hits a dichroic beam splitter that reflects wavelengths shorter than ' nm to the wavefront sensor leg consisting of an off-axis parabolic focusing mirror, a turning mirror, a collimating lens, the lenslet array, a set of relay lenses, and finally the wavefront sensor camera. Light at wavelengths longer than 600 $\mathrm{nm}$ passes through the dichroic beam splitter to the science camera leg, consisting of an off-axis parabolic focusing mirror, a turning mirror that sends the light to the back side of the optical bench, (B) a turning mirror that receives the light from the front side of the optical bench, and a dichroic beam splitter that transmits wavelengths longer than $900 \mathrm{~nm}$ to a turning mirror and thence to an infra-red science camera. Wavelengths shorter than $900 \mathrm{~nm}$ are reflected from the dichroic beam splitter to a turning mirror. A variable beam splitter after the turning mirror passes a selectable fraction of the light to the tip-tilt sensor and reflects the rest to a beam splitter cube. Half the light from this cube goes to a CCD scoring camera, and the other half to a low-speed wavefront sensor. The latter is used to monitor focus and other slowly varying aberrations introduced by the laser guide star. 


\section{SYSTEM DESCRIPTION}

The Lick Observatory adaptive optics system (2) is mounted at the $\mathrm{f} / 17$ Cassegrain focus of the $3 \mathrm{~m}$ Shane Telescope (12). The system can feed both an optical 1024×1024 CCD camera and a near-IR 256×256 NICMOS III camera. An optical schematic is shown in Figure 1.

The deformable mirror, built at LLNL, corrects wavefront phase over the telescope pupil. This thin glass mirror has 127 electro-restrictive (PMN) actuators arranged in a triangular pattern, each capable of deforming the front surface by up to \pm 4 $\mu \mathrm{m} ; 61$ of the actuators are actively controlled. A separate flat fast-steering mirror, built by Physik Instrumente, is used to correct the overall wavefront tip-tilt (image motion).

The Shack-Hartmann wavefront sensor has 37 subapertures in the clear aperture of the telescope. A Shack-Hartmann sensor divides the telescope pupil into subapertures using a set of miniature lenses (a lenslet array) placed in a reimaged pupil plane. The average wavefront slope in each subaperture is determined by measuring the position of the focused image formed by each lenslet. The constraint of continuity can then be imposed, and an estimate of the wavefront phase can be reconstructed, for example, from a least-squares fit. The subapertures have a diameter of $44 \mathrm{~cm}$ mapped to the primary mirror, and are arranged

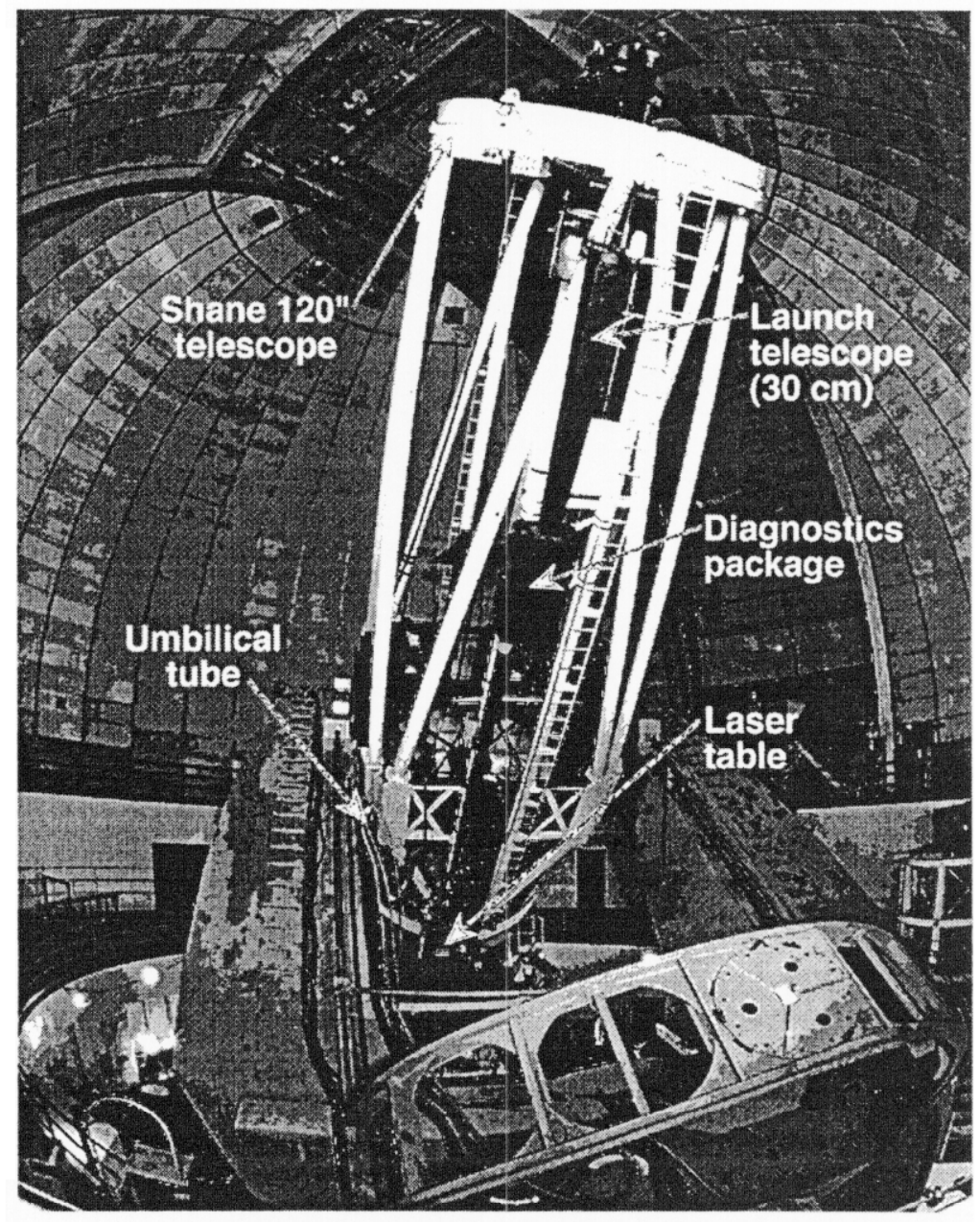

Figure 2. LLNL laser guide star installation at Lick Observatory. The dye master oscillator and the Nd:YAG pump lasers are in a room below the dome floor. An umbilical tube from this room to the telescope contains the lines that carry the dye, as well as optical fibers carrying high-power green YAG light and low-power yellow light from the dye master oscillator to the dye laser amplifier on the telescope. High-power yellow light from the dye laser amplifier travels up through the diagnostics package that monitors beam pointing and wavefront quality, and propagates into the sky through the launch telescope. 
in a triangular pattern to match the deformable mirror. The wavefront sensor, built by Adaptive Optics Associates, uses a $64 \times 64$ Lincoln Laboratory CCD, with read noise of $7 \mathrm{e}^{-}$per pixel at 1200 frames $\mathrm{s}^{-1}$.

A separate tip-tilt sensor is used in laser guide star mode. Because the laser travels up through the turbulent atmosphere before forming the guide star, the instantaneous physical position of the guide star is variable. This upgoing beam wander results in a difference between the apparent instantaneous positions of a laser guide star and a natural star, rendering the laser guide star unsuitable as a reference for stabilization of the overall image position of astronomical objects. The solution is to use a faint natural star as overall tip-tilt reference. If this technique is employed, the sky coverage for a laser guide star adaptive optics system is limited by the availability of suitable tip-tilt reference stars $(13,14)$. Since the requirements for a tip-tilt reference star are less severe than for a high-order wavefront reference beacon, use of a laser guide star significantly increases the sky coverage fraction for adaptive optics systems. The tip-tilt sensor, built at LLNL, uses 4 photon-counting avalanche photo-diodes as a quad cell.

The wavefront control computer is a 160 Mflop Mercury VME system with 4 Intel i860 processors. It is operated at a sample rate up to $500 \mathrm{~Hz}$ with control bandwidth $(0 \mathrm{~dB}$ crossover) up to $30 \mathrm{~Hz}$, including camera integration and readout rate as well as compute rate and transfer rate to the deformable mirror drivers. In laser guide star mode, the average tip-tilt from the wavefront sensor is utilized by a separate digital processor to control a high-bandwidth steering mirror within the laser, stabilizing the laser guide star on the wavefront sensor. The fast-steering mirror in the adaptive optics system is controlled at a bandwidth up to $120 \mathrm{~Hz}$ by a dedicated analog system using the signal from the avalanche photo-diode tip-tilt sensor.

The laser guide star system $(15,16)$ is based on a tunable dye laser pumped by flash-lamp-pumped frequency-doubled Nd:YAG lasers. Pump lasers are located in a room below the telescope dome and fiber-optically coupled to the dye laser and launch telescope on the side of the $3 \mathrm{~m}$ telescope. The dye laser produces light tuned to $589 \mathrm{~nm}$ which is projected into the sky by a refractive launch telescope with a $30 \mathrm{~cm}$ primary lens (17). Figure 2 shows the laser guide star hardware on the telescope. The laser projects $18 \mathrm{~W}$ of average power with pulse width of $\sim 100 \mathrm{~ns}$ and pulse repetition rate of $\sim 11 \mathrm{kHz}$.

The apparent size of the guide star in the sodium layer was measured in $10 \mathrm{sec}$ exposures to be as small as 1.8 arc seconds, with size determined by seeing on the up- and down-link paths. In both Sept. and Nov. 1996 the return signal from the laser guide star was comparable to that for a natural star with magnitude $7.0 \mathrm{in} \mathrm{V}$ band (a filter centered at $555 \mathrm{~nm}$, width $\sim 100$ $\mathrm{nm}$ ). This measured return signal is a factor of 2 higher than measured at LLNL in 1992 (9) for comparable laser power, and a factor of $\sim 4$ higher than previous measurements at Lick. Changes in return flux are expected due to seasonal variation in column density of the sodium layer.

\section{IMAGE IMPROVEMENT}

Figures $3 \mathrm{~A}$ and $\mathrm{B}$ show images at a wavelength of $2.2 \mu \mathrm{m}$ of the field star $\gamma$ Trianguli (magnitude $V=4.0$ ). In each image pair, the left image shows the star without any correction. In panel A the right image shows the effect of correction by the tip-tilt system alone. In panel B the right image shows the joint effect of correction by the tip-tilt system and the high-order adaptive optics system, using the natural star as a reference. Panel C shows two images of the field star SAO 56102 (V = 9.1): the left image is without any correction; the right image shows the joint effect of correction by the tip-tilt system and the high-order adaptive optics system with the laser guide star as a reference. All these images were recorded within an hour of each other on Oct. 1, 1996.

The measured value of the atmospheric coherence length $\mathrm{r}_{0}$ was $10.7 \mathrm{~cm}$ at $0.55 \mu \mathrm{m}$. In this test both the laser guide star and the field star were on-axis. Light from the field star was attenuated on the wavefront sensor using a narrow-band filter centered at $589 \mathrm{~nm}$. The field star was used as the tip-tilt guide star. Exposures lasted for $20 \mathrm{~s}$ for $\gamma$ Trianguli and $60 \mathrm{~s}$ for SAO 56102. The uncorrected image for $\gamma$ Trianguli has a normalized peak intensity $18 \%$ larger than that for SAO 56102, due to the difference in exposure times and to minor changes in calibration and atmospheric conditions. These images were made at about $5^{\circ}$ from zenith with adaptive optics sampling frequency of $200 \mathrm{~Hz}$.

Relative to the uncorrected images on the left side of Figure 3, the image peak intensity was increased by a factor of 1.4 for tip-tilt correction alone, by 4.2 for tip-tilt with high-order natural guide star adaptive optics, and by 3.3 for tip-tilt with highorder laser guide star adaptive optics. The FWHM was decreased by a factor of 1.3 (from 0.8 to 0.6 arc sec) in case of tip-tilt correction alone, and by a factor of 2.7 (to $0.3 \mathrm{arcsec}$ ) for both natural and laser guide star correction. Compared with tip-tilt correction alone, laser guide star adaptive optics increased the peak intensity by a factor of 2.4 and decreased the FWHM by a factor of 2 . 


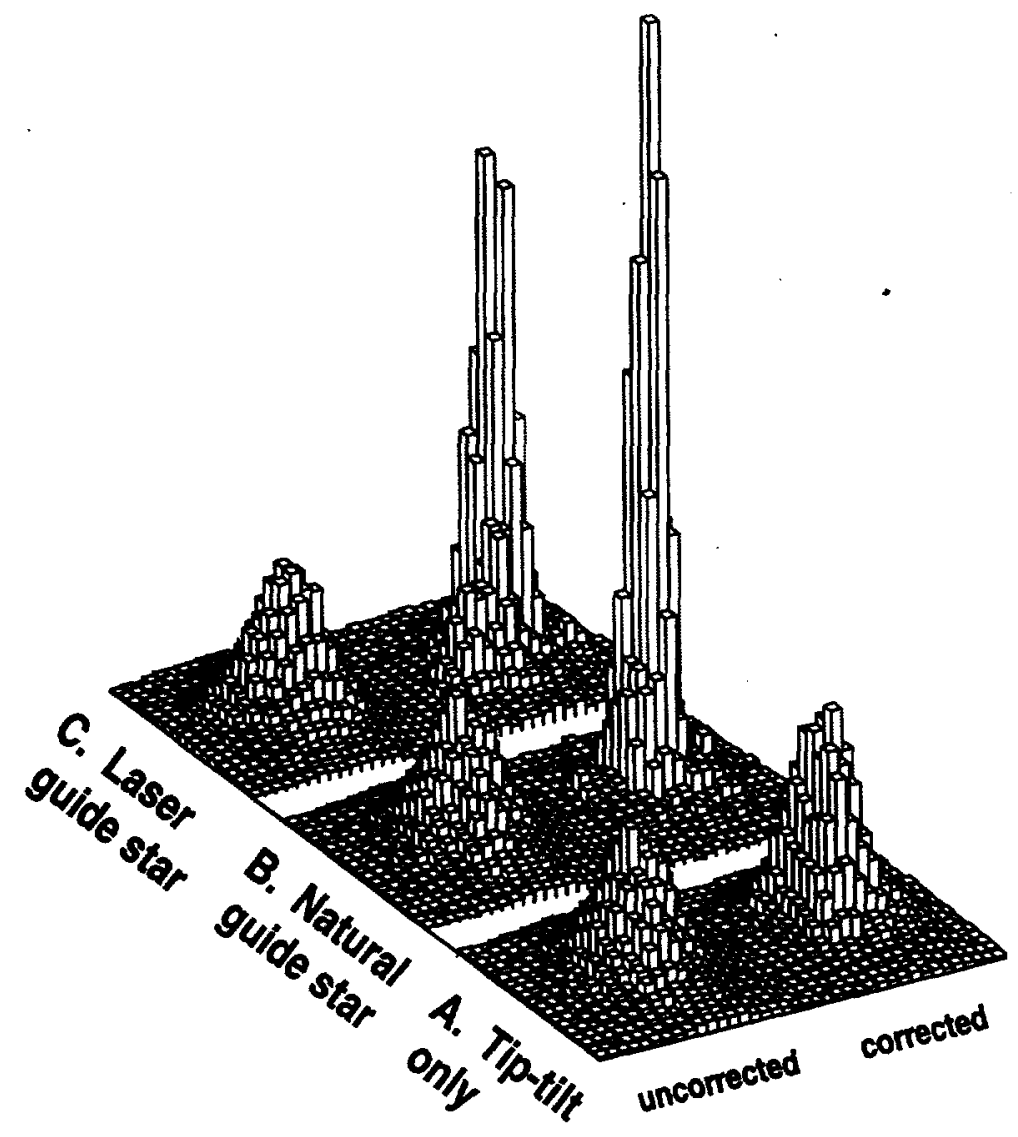

Figure 3. Image data without (left) and with (right) correction using (A) the tip-tilt system alone, (B) both the tiptilt and high-order adaptive optics system with a natural guide star, and $(C)$ both the tip-tilt and high-order adaptive optics system with the sodium-layer laser guide star. These images of the field stars (A, B) $\gamma$ Trianguli (20 s exposures) and (C) SAO 56102 (60 s exposures) were recorded within an hour of each other on October 1, 1996 at a wavelength of $2.2 \mu \mathrm{m}$. The image peak intensities after correction by adaptive optics are (A)1.4, (B) 4.2, and (C) 3.3 times the respective uncorrected values. The corrected image widths are (A) 0.6 and (B,C) 0.3 arc seconds, a factor of (A) 1.3 and $(B, C) 2.7$ less than the uncorrected size of 0.8 arc seconds. The increase in peak intensity using the laser guide star is $75 \%$ of that using the natural guide star, with other system parameters (including tip-tilt) held constant. [Note that the uncorrected image for $\gamma$ Trianguli in (A, B) has a normalized peak intensity $18 \%$ higher than that for SAO 56102 in (C)].

For the measured seeing conditions ( 0.8 arc sec at $\lambda=2.2 \mu \mathrm{m}, \mathrm{r}_{0}=10.7 \mathrm{~cm}$ at $\left.\lambda=0.55 \mu \mathrm{m}\right)$, direct integration of the modulation transfer function for telescope and ensemble-averaged atmosphere $(18,19)$ shows that the expected increase in peak intensity for tip-tilt correction is a factor of 3.7. However, taking into account the measured and inferred calibration errors of $1.7 \mathrm{rad}^{2}$ (assuming they have roughly the same spectrum as the tilt-corrected atmosphere) the expected intensity increase is only a factof 1.7 . The measured value was 1.4 .

Similar image improvement was obtained for several different stars on Sept. 30 and Oct. 1 as well as on Nov. 26 and 27, 1996, the latter under seeing conditions worse by a factor of 2-3, with surface winds of 30-40 mph. These nights showed little temperature variation, and we measured no significant contribution due to correction of primary mirror aberrations.

The encouraging nature of our results is best illustrated by the fact that the increase in peak intensity with laser guide star adaptive optics is $75 \%$ of the increase for natural guide stars, with all other system parameters including tip-tilt held constant. The corrected FWHM was identical for the laser and natural guide stars. In other words, high-order correction using the laser guide star is nearly as good as that using a natural star. 


\section{ERROR ANALYSIS}

The Strehl is a measure of image quality: the ratio of the measured peak intensity to that of a theoretical image limited only by diffraction. Table 1 shows that the Strehl of the laser guide star corrected image is $0.091,65 \%$ of that measured for the natural guide star corrected image. Ten percent of this difference is due to poorer performance of the tip-tilt system (discussed further below). Additional wavefront errors specific to the laser, due to finite size and ellipticity of the laser spot and uncertainty in sodium-layer height, are not large.

Table 1. Residual errors for adaptive optics correction using both a natural and laser guide star.

\begin{tabular}{lcc}
\hline & $\begin{array}{c}\text { Natural guide star } \\
\text { adaptive optics } \\
\text { Strehl ratio }\end{array}$ & $\begin{array}{c}\text { Lasec guide star } \\
\text { adaptive optics } \\
\text { Strehl ratio }\end{array}$ \\
\hline Measured Strehl from corrected image & 0.14 & 0.091 \\
Measured Strehl from uncorrected image & 0.033 & 0.028 \\
Predicted or measured Strehl from & & \\
individual wavefront error sources: & & \\
1. Fitting error & 0.82 & 0.82 \\
2. Servo error & 0.81 & 0.89 \\
3. Measurement error & 0.98 & 0.95 \\
4. Tip-tilt error & 0.76 & 0.66 \\
5. Calibration errors: & 0.89 & 0.89 \\
5.1. Internal to AO system & 0.52 & 0.57 \\
5.2. Internal to IR camera & $\mathrm{n} / \mathrm{a}$ & 0.98 \\
6. Predicted cone effect & & 0.23 \\
Total predicted Strehl after correction & 0.23 & 0.6 \\
Inferred internal calibration drift & & 0.65 \\
Inferred laser guide star calibration error & 0.6 & \\
\hline
\end{tabular}

The power spectrum of wavefront phase fluctuations measures the power per unit frequency interval present in residual uncorrected phase aberrations. The open and closed-loop phase power spectra are shown in Figure 4 for $(A)$ the natural guide star and (B) the laser guide star. Qualitative features of these power spectra are similar for laser- and natural-guide-star corrections. The upper curves in each panel represent measurements made with no correction; the lower curves are the residuals after adaptive optics and tip-tilt correction. Both the natural and laser guide star panels show that adaptive optics strongly decreases residual phase fluctuations at low frequencies, with the correction losing its impact at frequencies above 5$10 \mathrm{~Hz}$. The noise floor (the flat part of the power spectra on the far right of Figure 4) is due to the fact that above a certain frequency, the noise is larger than the signal. The power spectrum noise floor for the laser guide star is higher than for the natural guide star, because the laser spot is larger and dimmer than the natural star. This is reflected in Table 1 in the increased measurement error for the laser.

Each power spectrum was calculated from a series of 4096 wavefront sensor centroid measurements at a sampling frequency of $200 \mathrm{~Hz}$. The control bandwidth $(0 \mathrm{db}$ crosscver) is $\sim 10 \mathrm{~Hz}$ for laser and natural guide stars. This measures the highest speed at which the control loop can correct phase aberrations. From the measured open-loop power spectra, it can be inferred that the atmospheric coherence time $t_{0}$ was between 250 and $500 \mathrm{msec}$ for these observations, depending on the turbulence model.

A decomposition of the Strehl ratio into six contributing sources of residual aberration (20) is shown in Table 1. "Fitting error" is due to limitations on the ability of the deformable mirror to respond to high-spatial-frequency variations of the atmosphere, because of the finite number of actuators. "Servo error" is due to the finite bandwidth of the control loop. "Measurement error" is due to the finite ability of the wavefront sensor to measure the reference wavefront in the presence of noise. "Tip-tilt error" (tip-tilt servo- plus measurement-error) represents the finite ability of the tip-tilt system to stabilize image motion. "Calibration error" is due to residual non-common-path aberration between the wavefront sensor and science camera focal planes. "Cone effect" measures the difference between the wavefront measured from the finite-altitude laser guide 


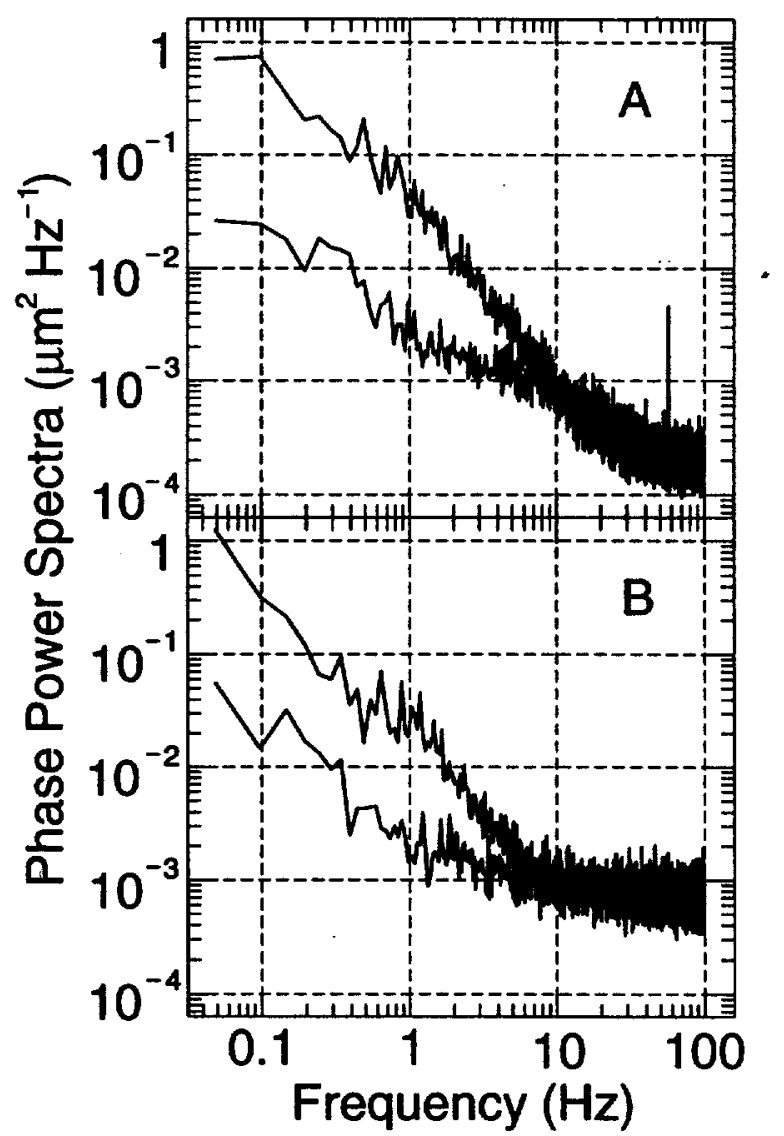

Figure 4. Reconstructed phase power spectra for (A) the bright natural star, $g$ Trianguli, and (B) the sodium-layer laser guide star. In both cases, the upper curve was calculated from data taken with the control loop open;the lower curve was calculated from data with the control loop closed (October 1, 1996). Laser guide star data were recorded at approximately the same time as the images shown in Figure 3; natural star data were recorded about $1 / 2$ hour earlier. Because the Hartmann sensor used $4 \times 4$ pixels ( 1.8 arc sec per pixel or 7.2 arc sec width of the quad cell overall) for its centroid determination, the dynamic range of the wavefront sensor was more than enough to accommodate the open-loop measurements, which showed rms. image motion of about 1 arc sec.

star and from a real star located an "infinite" distance away. Where appropriate, the Strehl ratios given in Table 1 are approximated by $\exp \left(-\sigma^{2}\right)$ where $\sigma$ is the rms wavefront error in radians at $2.2 \mu \mathrm{m}$.

The predicted fitting error was estimated using a standard scaling law from atmospheric turbulence theory (21) with atmospheric coherence length estimated from the uncorrected image FWHM. Measurement and servo errors were determined directly from measured wavefront phase power spectra. The measurement error was estimated from the integral of the power spectrum noise floor, taking into account filtering of the noise by the control loop. The factor by which the rms noise is reduced due to averaging by the control loop was estimated to be $\chi \doteq\left[(2 / \kappa) \tan ^{-1}(\kappa / 2)\right]^{1 / 2}$, where $\kappa$ is the ratio of the sampling frequency to the control bandwidth (13). Because the total measurement error is quite small for these experiments, finite laser guide star spot size is not a significant factor in our measurement error. Apart from the impact of finite laser spot size on measurement error (negligible in our experiments because of the very high signal to noise ratio), anisoplanatism due to finite laser spot size (22) contributes a mean square error of $\sigma^{2}=\left(0.1014 \theta_{\mathrm{gs}} / \theta_{0}\right)^{5 / 3}<0.001$ for the parameters of our experiment, where $\theta_{\mathrm{gs}}=1.8 \mathrm{arc} \mathrm{sec}$ is the laser spot size in the mesosphere and $\theta_{0}=15-20 \mathrm{arc} \mathrm{sec}$ is the isoplanatic angle at $2.2 \mu \mathrm{m}$. Hence, finite laser spot size is not a significant factor in our data. Servo error was calculated from the integral of the power spectrum after removal of the noise floor.

The tip-tilt errors in Table 1 are derived from measured quantities. For the natural guide star, tip-tilt error is calculated from measured power spectra of wavefront sensor centroid positions averaged over the pupil, after taking into account the intrinsic 
measurement error of the average centroid position. This cannot be done for the laser guide star because the average centroid position is affected by the deviation of the laser beam as it goes up through the atmosphere. Hence the tip-tilt measurement error for the laser was calculated by assuming the same servo error as for the natural guide star, and scaling the measurement error by the measured decrease in the sum voltage signal on the tip-tilt sensor quad cell. At least part of the degraded tip-tilt performance for the laser case is attributable to misalignment of the tip-tilt sensor, which has subsequently been corrected.

Residual error in calibration of non-common path optics arises from the adaptive optics system, and from inside the IR camera dewar. Calibration error for the adaptive optics system was measured using a white-light reference source and a CCD camera with a narrow-band filter centered at $700 \mathrm{~nm}$. Calibration error for the IR camera was measured using the same whitelight source and two different filters within the IR camera. The filter with narrower bandwidth, used for the brighter natural guide star, had slightly more measured calibration error. Calibration error was minimized by adjusting the deformable mirror to produce the best reference image.

For both laser and natural guide stars, the measured Strehl was about 0.6 that predicted from the measured error sources. This implies that there was a secular drift in calibration over the 6 hour interval between the last calibration and image data collection. This drift is due to flexure from temperature effects and other mechanical stresses, as well as inaccuracies in repositioning the wavefront sensor between the focal positions for the natural guide star and for the laser star. Errors such as these can be reduced by more frequent calibration.

\section{FUTURE PLANS}

We envision several upgrade paths for future improved performance. The two largest contributors to residual wavefront errors are calibration internal to the IR camera $(S=0.57)$, and tip-tilt error $(S=0.66)$. Additional laser guide star calibration error $(0.65)$ has been inferred by comparing laser and natural guide star results. The current IR camera may be improved with better alignment of the internal optics or with the fabrication of new optics. In addition, a new diffraction-limited IR camera is currently under construction. We are also investigating the technique of phase diversity (23) to directly measure the aberrations in the IR camera. This information could then be used to adjust the shape of the deformable mirror in order to optimize the image made by the IR camera.

To improve tip-tilt performance, the sensor has been realigned and the optical path to the tip-tilt sensor (Figure 1) is being reconfigured for higher throughput. With these new optics, limiting tip-tilt magnitudes for laser guide star correction are predicted to be $m_{\mathrm{V}}=14-15$ at Lick Observatory. For the laser guide star, the main limitation on image improvement for a science object comes from the need for a sufficiently bright tip-tilt reference. If the science object is point-like, it can be used as a tip-tilt reference itself. In that case it must be brighter than $\mathrm{mV}=14-15$ for Lick, or $\mathrm{mV}=19$ for the $10 \mathrm{~m}$ Keck Telescopes. Alternatively a tip-tilt reference star of this magnitude must be located within a few 10's of arc seconds of the science object.

Improving laser guide star calibration is an important topic for future research. As a first step we plan to implement a second wavefront sensor in a low-speed control loop to correct for calibration errors specific to laser guide star operation. The latter include the difference in size and shape on the wavefront sensor between the laser guide star and a natural star, and focus error introduced if the height of the sodium layer is not perfectly sensed.

\section{CONCLUSION}

In summary, significant image improvement has been demonstrated for the first time with a sodium-layer laser guide star adaptive optics system. Peak image intensity was increased by a factor of 2.4 compared with tip-tilt correction alone; image FWHM was decreased by a factor of 2. With improved calibration for the IR camera and laser guide star spot shape, the sodium guide star technique is predicted to perform very well even for the largest telescopes. For example with a sodiumlayer laser guide star system, the $10 \mathrm{~m}$ Keck Telescopes should have limiting tip-tilt magnitude $\mathrm{mV}=19$ with angular resolution as good as 0.02 arc sec in the near IR (24).

\section{ACKNOWLEDGMENTS}

Work performed under the auspices of the U.S. Department of Energy by the Lawrence Livermore National Laboratory under Contract number W 7405-Eng-48. 


\section{REFERENCES}

1. H. W. Babcock, "Adaptive optics revisited",Science 249, pp. 253-257, 1990.

2. L. A. Thompson, "Adaptive optics in astronomy", Physics Today 47, pp. 24- 31, 1994.

3. J. M. Brase, J. An, K. Avicola, H. D. Bissinger, H. W. Friedman, D. T. Gavel, B. Johnston, C. E. Max, S. S. Olivier, R. Presta, D. A. Rapp, J. T. Salmon, and K. E. Waltjen, "Adaptive optics at Lick Observatory: System architecture and operations", Proc. SPIE 2201, pp. 474-488, 1994.

4. S. S. Olivier, J. An, K. Avicola, H. D. Bissinger, J. M. Brase, H. W. Friedman, D. T. Gavel, E. M. Johansson, C. E. Max, K. E. Waltjen, W. Fisher, and W. Bradford, "Performance of adaptive optics at Lick Observatory", Proc. SPIE 2201, pp. 1110-1120, 1994.

5. R. Q. Fugate, D. L. Fried, G. A. Ameer, B. R. Boeke, S. L. Browne, P. H. Roberts, R. E. Raune, G. A. Tyler, and $L$. M. Wopat, "Measurement of atmospheric wavefront distortion using scattered light from a laser guidestar", Nature 353, pp. 144-146, 1991.

6. D. Fried and J. F. Belsher, "Analysis of fundamental limits to artificial-guide-star adaptive-optics-system performance for astronomical imaging", J. Opt. Soc. Am. A11, pp. 277-287, 1994.

7. W. Happer, G. MacDonald, C. E. Max, and F. Dyson, "Atmospheric-turbulence compensation by resonant optical backscattering from the sodium layer in the upper atmosphere",J. Opt. Soc. Am. A11, pp. 263-276, 1994.

8. R. Foy and A. Labeyrie, "Feasibility of adaptive telescope with laser probe", Astron. \& Astrophys. 152, pp. L29-L31, 1985.

9. K. Avicola, J. M. Brase, J. R. Morris, H. D. Bissinger, J. M. Duff, H. W. Friedman, D. T. Gavel, C. E. Max, S. S. Olivier, R. W. Presta, D. A. Rapp, J. T. Salmon, and K. E. Waltjen, "Sodium-layer laser guide star experimental results", J. Opt. Soc. Am. A11, pp. 825-831, 1994.

10. R. A. Humphreys, C. A. Primmerman, L. C. Bradley, and J. Herrmann, "Atmospheric-turbulence measurements using a synthetic beacon in the mesospheric sodium layer", Opt. Lett. 16, pp. 1367-1369, 1991.

11. M. Lloyd-Hart, J. R. P. Angel, B. Jacobsen, D. Wittman, R. Dekany, D. McCarthy, E. Kibblewhite, B. Carter, W. Wild, and J. Beletic, "Adaptive optics experiments using sodium laser guide stars", Astrophys. J. 439, pp. 455-473, 1995.

12. S. S. Olivier, J. An, K. Avicola, H. D. Bissinger, J. M. Brase, H. W. Friedman, D. T. Gavel, C. E. Max, J. T. Salmon, and K. E. Waltjen, "Performance of laser guide star adaptive optics at Lick Observatory", Proc. SPIE 2534, pp. 26-37, 1995.

13. S. S. Olivier, C. E. Max, D. Gavel, and J. Brase, "Tip-tilt compensation: resolution limits for ground based telescopes using laser guide star adaptive optics", Astrophys. J. 407, pp. 428-439, 1993.

14. S. S. Olivier and D. T. Gavel, "Tip-tilt compensation for astronomical imaging", J. Opt. Soc. Am. A11, pp. 368-378, 1994.

15. H. Friedman, G. Erbert, T. Kuklo, T. Salmon, D. Smauley, G. Thompson, and N. Wong, "Design of a fieldable laser system for a sodium laser guide star", Proc. SPIE 2201, pp. 352-363, 1994.

16. H. W. Friedman, G. V. Erbert, T. C. Kuklo, J. G. Malik, J. T. Salmon, D. A. Smauley, G. R. Thompson, and N. J. Wong, "Sodium beacon laser system for the Lick Observatory", Proc. SPIE 2534, pp. 150-160, 1995.

17. H. W. Friedman, G. V. Erbert, D. T. Gavel, T. C. Kuklo, J. G. Malik, J. T. Salmon, D. A. Smauley, G. R. Thompson, "A sodium guide star laser system for the Lick Observatory 3 meter telescope", Proc. European Southern Observatory, Conference no. 54, (ESO, Garching, Germany), pp. 207-209, 1995.

18. D. L. Fried, "Optical resolution through a randomly inhomogeneous medium for very long and very short exposures", J. Opt. Soc. Am. 56, pp. 1372-1379, 1966.

19. D. T. Gavel and S. S. Olivier, "Simulation and analysis of laser guide star adaptive optics systems for the 8- to 10-meter-class telescopes", Proc. SPIE 2201, pp. 295-303, 1994.

20. For a review see J. M. Beckers, "Adaptive optics for astronomy - principles, performance, and applications", Ann. Rev. Astron. Astrophys. 31, pp. 13-62, 1993.

21. D. L. Fried, "Statistics of a geometric representation of wavefront distortion", J. Opt. Soc. Am. 55, pp. 14271435, 1965.

22. R. Sasiela, "A unified approach to electromagnetic wave propagation in turbulence and the evaluation of multiparameter integrals", Lincoln Laboratory Technical Report no. 807, (MIT Lincoln Lab, Lexington, MA), 1988

23. R. A. Gonsalves, "Phase retrieval and diversity in adaptive optics", Opt. Eng. 21, pp. 829-832, 1982.

24. C. E. Max, D. T. Gavel, S. S. Olivier, and J. Brase, "Limitations and expected performance of adaptive optics at Keck", chapter 4 in Adaptive Optics for the Keck Observatory (W. M. Keck Observatory, Waimea, HI), report. no. 208, 146 pages, 1995. 


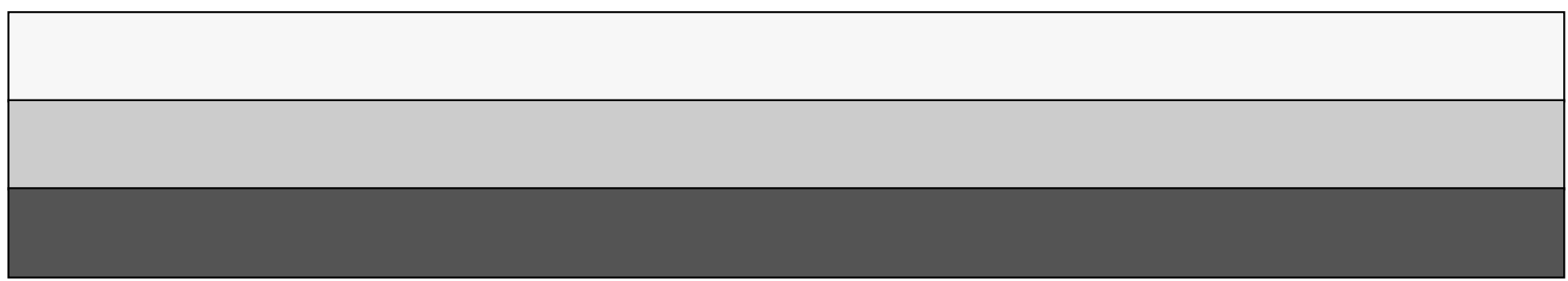

